\title{
Cortical activation patterns in healthy subjects during the traditional Japanese word generation task Shiritori determined by multichannel near-infrared spectroscopy
}

\author{
Takayuki Nakahachi ${ }^{1,2}$, Ryouhei Ishii ${ }^{1 *}$, Leonides Canuet ${ }^{3}$, Hidetoshi Takahashi ${ }^{2}$, Makoto Ishitobi ${ }^{2}$, Yoko Kamio ${ }^{2}$ \\ and Masao Iwase ${ }^{1}$
}

\author{
* Correspondence: \\ ishii@psy.med.osaka-u.ac.jp \\ 'Department of Clinical \\ Neuroscience and Psychiatry, Osaka \\ University Graduate School of \\ Medicine, D3 2-2 Yamadaoka, Suita, \\ Osaka 565-0871, Japan \\ Full list of author information is \\ available at the end of the article
}

\begin{abstract}
Background: Shiritori is a traditional Japanese game based on word generation to letters (WGL). This game is familiar to most Japanese people, young and old. Previous functional neuroimaging studies using magnetoencephalography and functional magnetic resonance imaging suggested that compared to conventional WGL, Shiritori could activate broader cortical areas, including the frontal lobe and the superior temporal cortex in healthy subjects. These studies were conducted under restricted experimental conditions without overt speech, that prevented the subjects from performing naturally the Shiritori task. The present study aims to determine cortical activation patterns in healthy subjects during the Shiritori task with overt speech.
\end{abstract}

Methods: Twenty-five healthy Japanese native speakers were investigated using multichannel near-infrared spectroscopy, which is not sensitive to motion artifact caused by overt speech and is able to cover frontal and superior temporal areas.

Results: Significant hemodynamic responses were widely distributed over the frontal and superior temporal cortex bilaterally. The activity could be divided into four clusters, with the channels in each cluster showing similar hemodynamic responses, one of which appears to correspond to the frontal and temporal language-related networks in the brain.

Conclusions: Cognitive functions subserved by fronto-temporal regions are elicited by Shiritori task with overt speech in healthy subjects. Thus, Shiritori might potentially be used as a clinically-relevant task to activate language-related networks in the brain.

Keywords: Frontal cortex, Hemodynamic responses, Near-infrared spectroscopy, Shiritori, Superior temporal cortex, Word generation to letters

\section{Background}

In the previous study using magnetoencephalography (MEG) that is a neuropsychiatric electrophysiological technique, our research group determined cortical activation patterns in healthy subjects during Japanese popular word generation task "Shiritori" without overt speech [27]. In the present study, we investigated whether cortical

(c) 2016 Nakahachi et al. Open Access This article is distributed under the terms of the Creative Commons Attribution 4.0 International License (http://creativecommons.org/licenses/by/4.0/), which permits unrestricted use, distribution, and reproduction in any medium, provided you give appropriate credit to the original author(s) and the source, provide a link to the Creative Commons license, and indicate if changes were made. The Creative Commons Public Domain Dedication waiver (http://creativecommons.org/ publicdomain/zero/1.0/) applies to the data made available in this article, unless otherwise stated. 
activation patterns in healthy subjects during Shiritori with overt speech were detected as is the case in MEG by using near-infrared spectroscopy (NIRS), which is a comparatively widespread neuroimaging technique in more hospitals and research institutes than MEG because of its easy usability, high portability, low cost and little restriction of movement (for a review see [5]). This investigation is important for the development of clinical tests applying functional neuroimaging during Shiritori performance.

Shiritori (Shiri meaning "buttock" and Tori meaning "taking") is a kind of game related to word generation to letters (WGL), which is familiar to Japanese people of all ages. It has long been played for over a thousand years as a word chain game [22]. During Shiritori, the player must say a noun that starts with the last syllable of the previously said noun, making a word chain as long as possible (Fig. 1). Most Japanese syllables are open syllables consisting of a vowel or consonant-vowel sequence. Syllables at the beginning of Japanese nouns are occasionally also used as the ending. In addition, Japanese letters consist of "kana" and "kanji" characters, the former being phonograms in which character-to-sound correspondence is one-to-one and the latter logograms which shows a minimum linguistic unit having meaning. There are 48 kana characters (each with a different pronunciation and corresponding to one syllable, forming a syllabary) that can be used in WGL. Because so many Japanese people with a wide range of cognitive levels can play Shiritori willingly, needing only the instruction "Let's play Shiritori", this would make it clinically convenient to use: if it were found to activate broad cortical areas, it might have a potential to serve as a neuropsychological test that should induce a high level of compliance from wide range of Japanese patients to assess cortical activity as well as an evidence-based and motivating tool for use in cognitive neurorehabilitation programs.

Functional neuroimaging studies aiming to determine cortical activation patterns of Shiritori performance in healthy subjects, have provided evidence indicating that in addition to the predominant frontal lobe involvement commonly seen using conventional WGL tasks, the superior temporal cortex also shows regional activation [14, 27]. Yamamoto et al. [27] speculated that this finding might be attributed to the internal

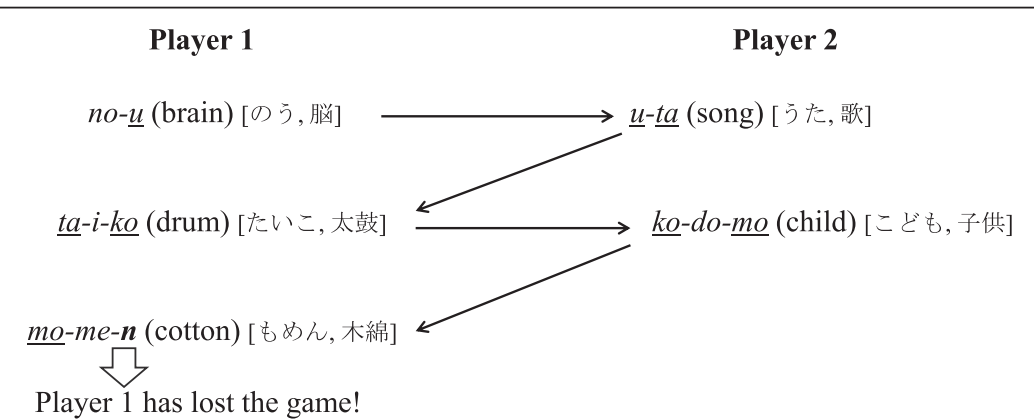

Fig. 1 How to play Shiritori. The illustration is an example of playing Japanese word chain game "Shiritori" in the case of two players, where the pronounced Japanese words are written in Romanized letters and segmented by hyphens into syllables, with the English translations shown in parentheses and the Japanese notation shown as [in kana character, in kanji character]. Say the player 1 starts the chain with the word no- $u$ with the cue syllable (underlined) $u$, the next player 2 could say $u$-ta, and the player 1 ta-i-ko, then the player 2 ko-do-mo, and the player 1 mo-me-n, which means the player 1 lost the game because the consonant $/ \mathrm{n}$ / is never used to begin Japanese nouns but does occasionally end them (i.e. it constitutes closed syllables exclusively) 
phonological processing needed in the Shiritori task for repeating and listening the last syllable of the previous noun in the subject's mind until the next noun is generated. The authors suggested that in Japanese subjects, Shiritori might be more suitable than conventional WGL for investigating language-related networks and fronto-temporal dysfunction like that seen in schizophrenia and other neuropsychiatric disorders. However, since these functional neuroimaging studies used magnetoencephalography [27] or functional magnetic resonance imaging (fMRI) [14], Shiritori was performed under restricted experimental conditions and without overt speech. It is difficult, therefore, to say that cortical activation patterns were determined during naturally performed Shiritori. Furthermore, behavioral performance (the number of generated words) associated with the subject's brain activity cannot be confirmed when the task is performed covertly.

NIRS is considered the best means of detecting cortical activity during the naturally performed WGL or verbal fluency test due to its low sensitivity to motion artifact by overt speech $[5,9,26]$. Although NIRS is a non-invasive functional brain imaging method, it is a powerful tool because near-infrared light do not only penetrate into tissues, but it is also differentially absorbed by hemoglobin $(\mathrm{Hb})$ depending on its oxygenation state and the optical path length in tissues (modified Beer-Lambert Law). The law is expressed as $A=\varepsilon C L+S$, where $A$ is the absorbance, $\varepsilon$ is the molar absorption coefficient, $\mathrm{C}$ is the chromophore concentration, $\mathrm{L}$ is the optical path length between emitter and detector of the NIRS's probe, and $\mathrm{S}$ is the optical attenuation associated with scattering. This relationship allows NIRS to detect relative changes in concentration of oxygenated hemoglobin $([\mathrm{oxy}-\mathrm{Hb}])$ and deoxygenated hemoglobin ([deoxy-Hb]) by emitting near-infrared light at some different wavelengths into the brain tissue and detecting its residue [6,12, 15]. Areas of high neural activity exhibit increased oxygen consumption as well as blood supply to ensure oxygenated hemoglobin provision (neurovascular coupling) ([8]; [13]). This means that neural activation is measured indirectly by relative changes in regional cerebral blood volumes (rCBV).

Recently, in schizophrenia Fujiki et al. [9] reported significantly reduced activation of bilateral prefrontal cortex compared to healthy subjects during the Shiritori task using multichannel NIRS, which was firstly applied to the Shiritori task in the English literature published internationally (this is described in the paper and also investigated by us). Although this study indicated usability of NIRS during Shiritori task performance with overt speech in detecting differences of activation in schizophrenia compared to healthy subjects, cortical activation patterns of healthy subjects themselves were not clarified. To our knowledge, this point has not been elucidated even in the Japanese literatures. Therefore, in the present study using multichannel NIRS with more measurement points than the previous study [9], we aimed to determine cortical activation patterns in healthy Japanese native speakers during Shiritori task performance with overt speech. This preliminary study would contribute to establish canonical referential data of Japanese healthy subjects for control group in future clinical studies. We hypothesized that significant activation would be widely detected across the frontal and superior temporal cortex during the Shiritori task, and that the activation could be divided into different clusters with distinctive features. 


\section{Methods}

\section{Subjects}

Twenty-seven healthy Japanese native speakers were participated in this study. The subjects were recruited from graduate and undergraduate students, clerks and their acquaintances in the universities related to the authors. They are confirmed to have no history of psychiatric or neurological disorders by an experienced neuropsychiatrist. Two subjects were excluded because of left-handedness and excessive motion artifacts respectively, leaving 25 right-handed subjects (13 men, 12 women; mean age $27.0 \pm$ 6.9 years).

Written informed consent was obtained from all subjects before participating in the experiments. All procedures and methods were in agreement with the policies and principles set forth in the Declaration of Helsinki. This study was approved by the Ethics Committee of Osaka University Graduate School of Medicine.

\section{NIRS measurement}

We measured relative changes in [oxy-Hb] and [deoxy-Hb]. Then, we calculated total hemoglobin ([total-Hb]) by combining these two measures based on multichannel NIRS (ETG-4000; Hitachi Medical Corporation, Tokyo, Japan) data acquired during the Shiritori task. The ETG-4000 uses two sorts of near-infrared light, $695 \mathrm{~nm}$ and $830 \mathrm{~nm}$. Seventeen laser diodes (emitters) and sixteen photodiodes (detectors) were mounted reciprocally at $3-\mathrm{cm}$ intervals on a piece of thermoplastic shell $(3 \times 11)$ attached to the frontal and superior temporal areas with adjustable straps. Detection depth was $2-3 \mathrm{~cm}$ under the skin surface in 52 separate areas $(6 \mathrm{~cm}$ high $\times 30 \mathrm{~cm}$ wide). The most ventral center photodiode was located on Fpz according to the international 10/20 system for electroencephalography. The channels at the most lateral posterior edges corresponded to the T3-Cz-T4 row of the international $10 / 20$ system or marginally anterior to this row (Fig. 2). This setting of ETG-4000 probe mounted with 52 channels covers the area including bilateral prefrontal, primary motor, supplementary motor, premotor, and superior temporal cortical surface regions $[24,26]$.

\section{Tasks}

In the Shiritori task, the examiner provided a single cue syllable for the first noun orally just before the task period, and the subject vocalized a noun beginning with this designated syllable, followed by vocalizing another noun beginning with the final syllable of the previous noun, and so on. The subject did this task by him or herself with the aim of producing as many different nouns as possible. If the subject produced a noun ending in $/ \mathrm{n} /$, which is a well-known rule of Shiritori meaning the word chain game is over (Fig. 1), the examiner would provide a different syllable to enable the subject to continue with the word game (this did not actually need to be used in the present study).

In the baseline task, subjects were instructed to stare blankly in front at a white wall trying, as far as possible, not to think. We applied this method of resting state as the base-line according to the previous functional neuroimaging studies using Shiritori $[14,27]$. 


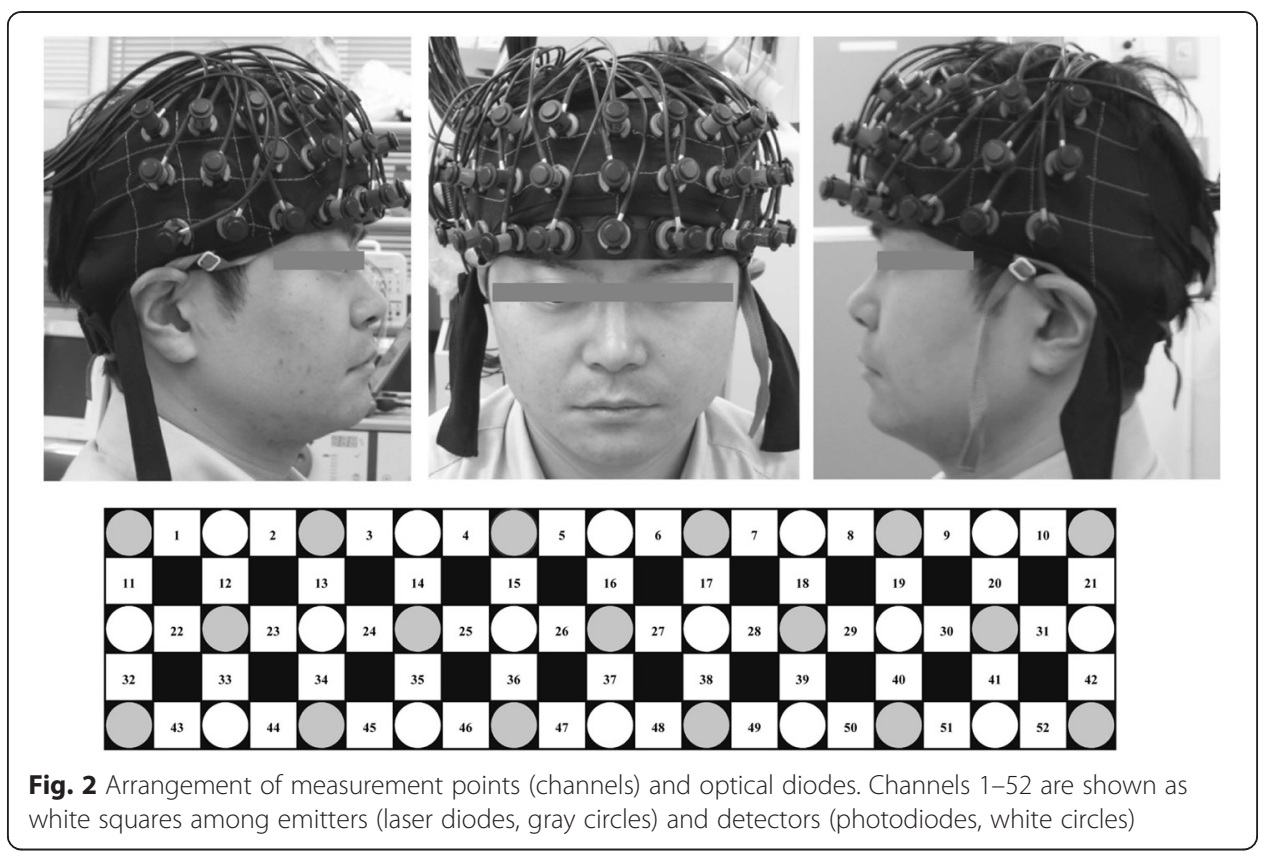

\section{Procedure}

During data collection, the subject sat on a chair in a silent room. The subject performed the first baseline task ( $30 \mathrm{~s}$ ), the Shiritori task (90 s), and the second baseline task (60 s). Instructions were given orally.

\section{Data analysis}

Changes in $[\mathrm{oxy}-\mathrm{Hb}]$ and [deoxy-Hb] are represented as the product of hemoglobin's concentration and optical path length, that produces a measurement of $\mathrm{mM} \cdot \mathrm{mm}$. We analyzed changes in [oxy-Hb] at temporal resolution of $100 \mathrm{msec}$. [oxy-Hb] is said to be most sensitive to changes in rCBV, and strongly correlates with the FMRI BOLD signal, while the direction of changes in [deoxy- $\mathrm{Hb}$ ] is influenced by changes in blood oxygenation and volume of the vein $[12,13,21]$. However, to give a fuller depiction of the data being collected with NIRS, grand average waveforms of changes in [deoxy-Hb] and [total-Hb] during the Shiritori task for all channels were superimposed on the waveforms of [oxy-Hb] (see Additional file 1).

The ETG-4000 analysis software was set at the "integral mode". In that configuration, mean changes in [oxy-Hb] during the baseline states $10 \mathrm{~s}$ before the start of the activation task period and after $50 \mathrm{~s}$ from the finish of the activation task period were corrected to $0 \mathrm{mM} \cdot \mathrm{mm}$ by using linear fitting. The activation task period was set at $90 \mathrm{~s}$, and the recovery period from the finish of activation task period up to baseline stabilization was set at $50 \mathrm{~s}$. To smooth out brief motion artifacts, we used the moving average method with a 5-s window. Mean changes in [oxy-Hb] during the Shiritori task for each channel were calculated for each subject, and followed by a two-tailed single-sample t-test. We previously succeeded in determining activation patterns during cognitive tasks in healthy subjects by using these setting and analysis with the ETG-4000 [17, 18]. 
Next, we divided all 52 channels into groups of channels showing similar changes in [oxy- $\mathrm{Hb}]$, using hierarchical cluster analysis. In the analysis, distances between the channels and between the clusters were measured by the squared Euclidean distance and the Ward method, respectively, using grand averaged data for every $0.1 \mathrm{~s}$ of changes in $[\mathrm{oxy}-\mathrm{Hb}]$ across all the channels. Moreover, we indicated the representative waveform for the each cluster based on the mean changes in [oxy-Hb] per $0.1 \mathrm{~s}$ of all the channels belonging to each cluster. Superimposed displays of these representative waveforms were made by segmenting the task period, and linear regressions were conducted to observe changes in them.

To determine the relationship between changes in [oxy-Hb] and Shiritori task performance, we calculated Pearson's correlation coefficients for each channel between the mean values of $[\mathrm{oxy}-\mathrm{Hb}]$ changes and the number of words generated during the Shiritori task.

The two-tailed single-sample t-test and correlation analysis were conducted on all 52 channels; therefore, the significant $\alpha$ levels of 0.05 were corrected using the false discovery rate (FDR) [2] to control for multiple comparisons, which can be applied to multichannel NIRS [4].

Finally, to determine the cortical laterality of changes in [oxy-Hb] during the Shiritori task, the average of the mean changes in [oxy-Hb] across all subjects were compared for the 25 respective channels of the left and right hemispheres at the symmetrical position using a paired, two-tailed Student's t-test, with significance set at $p=0.05$. Further, each subject's changes in $[\mathrm{oxy}-\mathrm{Hb}]$ during the Shiritori task were analyzed using the laterality analysis method described above. In this case, since paired $t$-tests were performed for 25 subjects, significant $\alpha$ levels of 0.05 were corrected using the FDR.

All statistical analysis was performed using SPSS software (version $21.0 \mathrm{~J}$ ).

\section{Results}

All the subjects in this study performed the Shiritori task without failure. The mean \pm standard deviation of the number of words generated during the Shiritori task for $90 \mathrm{~s}$ was $28 \pm 8$.

Next, the grand average waveform corresponding to changes in [oxy-Hb] across subjects was generated for each channel. To detect significant activation, mean changes in [oxy- $\mathrm{Hb}]$ during the Shiritori task were compared with those during the baseline task for all 52 channels. Forty-two channels $(80.8 \%)$ were shown to have significant increases in $[\mathrm{oxy}-\mathrm{Hb}]$ on a single-sample t-test with FDR ( $p=8.19 \mathrm{E}-09$ to 0.038$)$ (Table 1 , Fig. 3). Cluster analysis of the changes in [oxy-Hb] per $0.1 \mathrm{~s}$ of the grand average waveforms for all the channels divided these channels into four clusters. In Fig. 3, the channels included in different clusters are shown in different colors, and the distances among the clusters, indicating similarity of waveforms in channels belonging to an individual cluster, are depicted in a dendrogram. Representative waveforms, produced by averaging the changes in [oxy- $\mathrm{Hb}$ ] per $0.1 \mathrm{~s}$ across the channels included in each cluster, are shown as Clusters I to IV in descending order of the mean changes in [oxy-Hb] of the representative waveform: the yellow, blue, dark blue, and green clusters in Fig. 3 are denoted as Clusters I, II, III and IV, respectively. Observing these representative waveforms along the time course of the Shiritori task, the waveforms for all clusters rose linearly until $5 \mathrm{~s}$ after beginning the task, and thereafter all except the Cluster I 
Table 1 Individual channel values

\begin{tabular}{|c|c|c|}
\hline Right channel & Activation & $t(p$ value $)$ \\
\hline $\mathrm{CH} 1$ & $0.152 \pm 0.275$ & $2.761(0.011)^{a}$ \\
\hline $\mathrm{CH} 2$ & $0.108 \pm 0.240$ & $2.251(0.034)^{a}$ \\
\hline $\mathrm{CH} 3$ & $0.045 \pm 0.282$ & $0.796(0.434)$ \\
\hline $\mathrm{CH} 4$ & $0.077 \pm 0.186$ & $2.084(0.048)$ \\
\hline $\mathrm{CH} 5$ & $0.029 \pm 0.152$ & $0.954(0.350)$ \\
\hline $\mathrm{CH} 11$ & $0.203 \pm 0.274$ & $3.706(0.001)^{a}$ \\
\hline $\mathrm{CH} 12$ & $0.307 \pm 0.306$ & $5.016(<0.001)^{\mathrm{a}}$ \\
\hline $\mathrm{CH} 13$ & $0.157 \pm 0.281$ & $2.798(0.010)^{a}$ \\
\hline $\mathrm{CH} 14$ & $0.145 \pm 0.276$ & $2.628(0.015)^{a}$ \\
\hline $\mathrm{CH} 15$ & $0.072 \pm 0.182$ & $1.969(0.061)$ \\
\hline $\mathrm{CH} 22$ & $0.364 \pm 0.295$ & $6.164(<0.001)^{a}$ \\
\hline $\mathrm{CH} 23$ & $0.484 \pm 0.375$ & $6.447(<0.001)^{a}$ \\
\hline $\mathrm{CH} 24$ & $0.232 \pm 0.261$ & $4.434(<0.001)^{a}$ \\
\hline $\mathrm{CH} 25$ & $0.143 \pm 0.248$ & $2.881(0.008)^{a}$ \\
\hline $\mathrm{CH} 26$ & $0.122 \pm 0.236$ & $2.590(0.016)^{a}$ \\
\hline $\mathrm{CH} 32$ & $0.390 \pm 0.321$ & $6.060(<0.001)^{a}$ \\
\hline $\mathrm{CH} 33$ & $0.377 \pm 0.302$ & $6.245(<0.001)^{a}$ \\
\hline $\mathrm{CH} 34$ & $0.386 \pm 0.286$ & $6.750(<0.001)^{a}$ \\
\hline $\mathrm{CH} 35$ & $0.270 \pm 0.281$ & $4.817(<0.001)^{a}$ \\
\hline $\mathrm{CH} 36$ & $0.192 \pm 0.260$ & $3.688(0.001)^{a}$ \\
\hline $\mathrm{CH} 43$ & $0.415 \pm 0.414$ & $5.014(<0.001)^{a}$ \\
\hline $\mathrm{CH} 44$ & $0.500 \pm 0.290$ & $8.621(<0.001)^{a}$ \\
\hline $\mathrm{CH} 45$ & $0.476 \pm 0.288$ & $8.256(<0.001)^{a}$ \\
\hline $\mathrm{CH} 46$ & $0.243 \pm 0.316$ & $3.844(0.001)^{\mathrm{a}}$ \\
\hline $\mathrm{CH} 47$ & $0.216 \pm 0.253$ & $4.266\left(<0.001^{a}\right)$ \\
\hline Left channel & Activation & $t(p$ value $)$ \\
\hline $\mathrm{CH} 6$ & $0.048 \pm 0.162$ & $1.486(0.150)$ \\
\hline $\mathrm{CH} 7$ & $0.072 \pm 0.185$ & $1.952(0.063)$ \\
\hline $\mathrm{CH} 8$ & $0.101 \pm 0.229$ & $2.196(0.038)^{a}$ \\
\hline $\mathrm{CH} 9$ & $0.037 \pm 0.276$ & $0.679(0.503)$ \\
\hline $\mathrm{CH} 10$ & $0.145 \pm 0.281$ & $2.583(0.016)^{a}$ \\
\hline $\mathrm{CH} 17$ & $0.108 \pm 0.199$ & $2.718(0.012)^{a}$ \\
\hline $\mathrm{CH} 18$ & $0.155 \pm 0.234$ & $3.308(0.003)^{a}$ \\
\hline $\mathrm{CH} 19$ & $0.165 \pm 0.262$ & $3.146(0.004)^{a}$ \\
\hline $\mathrm{CH} 2 \mathrm{O}$ & $0.172 \pm 0.490$ & $1.757(0.092)$ \\
\hline $\mathrm{CH} 21$ & $0.232 \pm 0.378$ & $3.065(0.005)^{a}$ \\
\hline $\mathrm{CH} 27$ & $0.133 \pm 0.291$ & $2.290(0.031)^{\mathrm{a}}$ \\
\hline $\mathrm{CH} 28$ & $0.164 \pm 0.268$ & $3.069(0.005)^{a}$ \\
\hline $\mathrm{CH} 29$ & $0.246 \pm 0.272$ & $4.529(<0.001)^{a}$ \\
\hline $\mathrm{CH} 30$ & $0.417 \pm 0.553$ & $3.768(-0.001)^{a}$ \\
\hline $\mathrm{CH} 31$ & $0.397 \pm 0.552$ & $3.597(0.001)^{a}$ \\
\hline $\mathrm{CH} 38$ & $0.114 \pm 0.357$ & $1.589(0.125)$ \\
\hline $\mathrm{CH} 39$ & $0.242 \pm 0.302$ & $4.011(0.001)^{a}$ \\
\hline $\mathrm{CH} 40$ & $0.342 \pm 0.341$ & $5.015(<0.001)^{a}$ \\
\hline
\end{tabular}


Table 1 Individual channel values (Continued)

\begin{tabular}{lll}
\hline $\mathrm{CH} 41$ & $0.438 \pm 0.408$ & $5.361(<0.001)^{\mathrm{a}}$ \\
$\mathrm{CH} 42$ & $0.484 \pm 0.388$ & $6.239(<0.001)^{\mathrm{a}}$ \\
$\mathrm{CH} 48$ & $0.234 \pm 0.363$ & $3.219(0.004)^{\mathrm{a}}$ \\
$\mathrm{CH} 49$ & $0.180 \pm 0.346$ & $2.606(0.016)^{\mathrm{a}}$ \\
$\mathrm{CH} 50$ & $0.461 \pm 0.323$ & $7.131(<0.001)^{\mathrm{a}}$ \\
$\mathrm{CH} 51$ & $0.496 \pm 0.419$ & $5.912(<0.001)^{\mathrm{a}}$ \\
$\mathrm{CH} 52$ & $0.497 \pm 0.353$ & $7.042(<0.001)^{\mathrm{a}}$ \\
Middle channel & Activation & $t(p$ value $)$ \\
$\mathrm{CH} 16$ & $0.046 \pm 0.222$ & $1.028(0.314)$ \\
$\mathrm{CH} 37$ & $0.160 \pm 0.309$ & $2.587(0.016)^{\mathrm{a}}$ \\
\hline
\end{tabular}

Activation, The average across 25 participants' mean values of changes in [oxy-Hb] $\pm \mathrm{SD}(\mathrm{mM} \mathrm{mm})$ during the Shiritori task in each channel; $t$, The $t$ values derived by comparing the 25 participants' mean changes in [oxy- $\mathrm{Hb}$ ] during the Shiritori task with the baseline using a single-sample $t$ test; ${ }^{a}$, Significance; $\mathrm{CH}$, Channel

waveform dropped for several seconds before rising again up to about $30 \mathrm{~s}$ after beginning the task. The linear rise in the Cluster I waveform that continued from the beginning also stopped around this time point. Then all representative waveforms dropped for several seconds, before showing a fluctuating slow rise to the end of the task period. Therefore, we segmented the task period at the two time points of 5 and $30 \mathrm{~s}$ after beginning the task and added the regression line for each representative waveform.

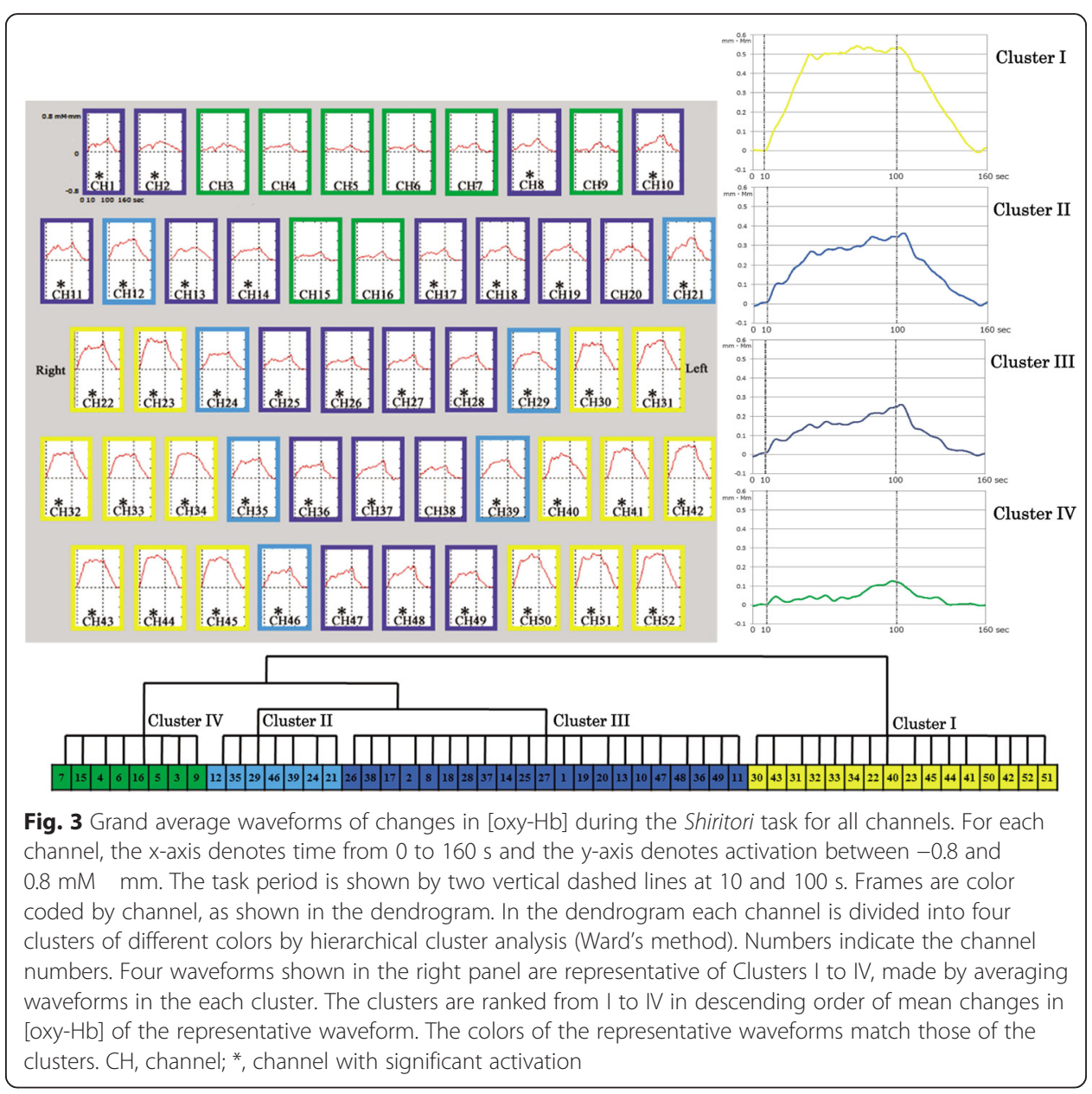


(a)

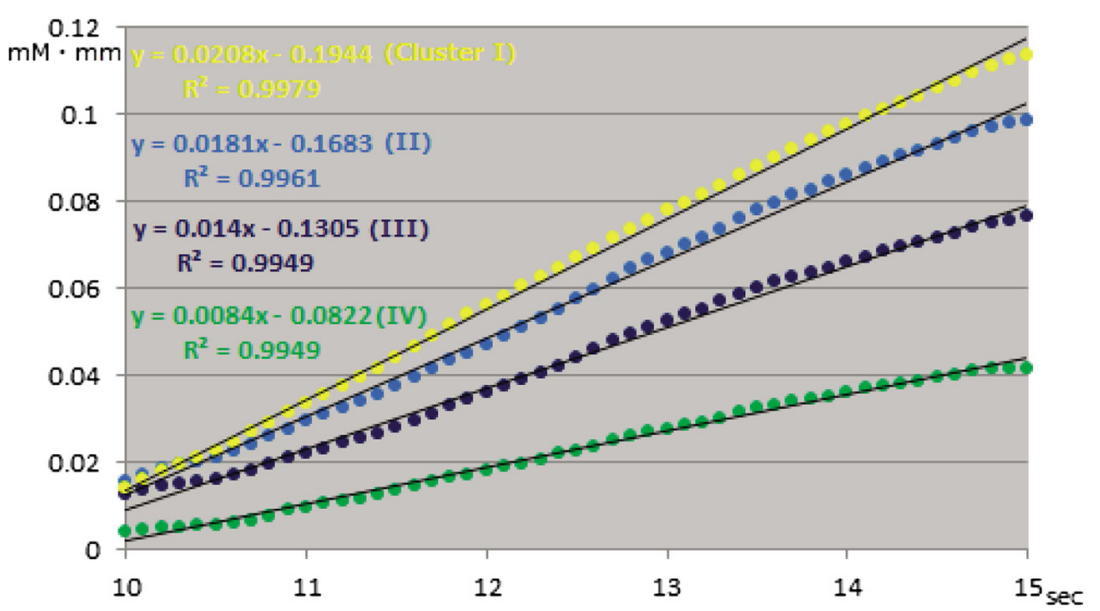

(b)

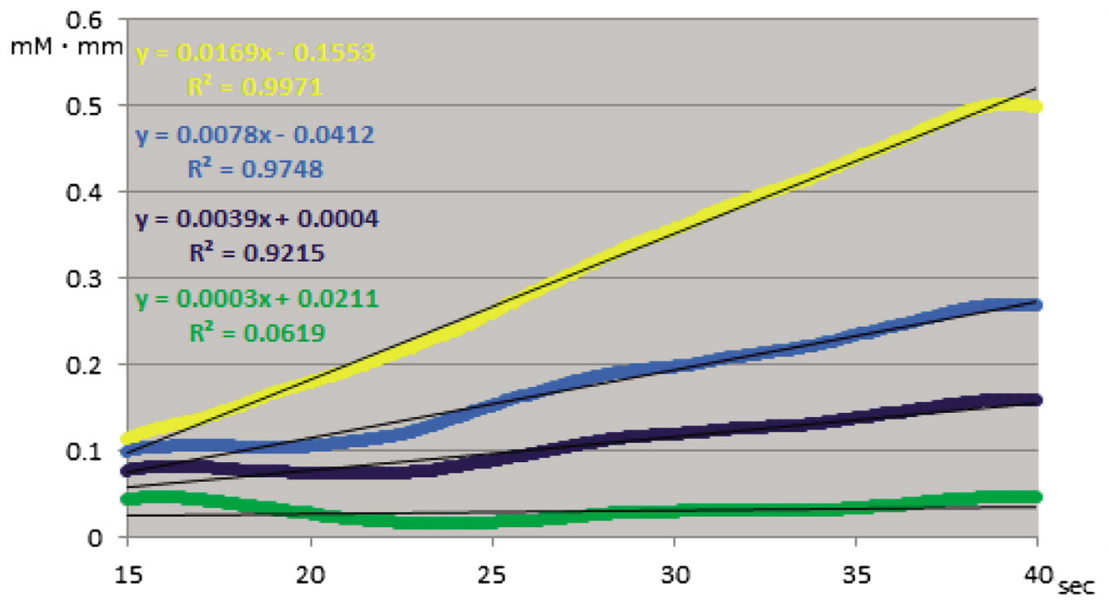

(c) $\mathrm{mM} \cdot \mathrm{0.6} \mathrm{mm}$

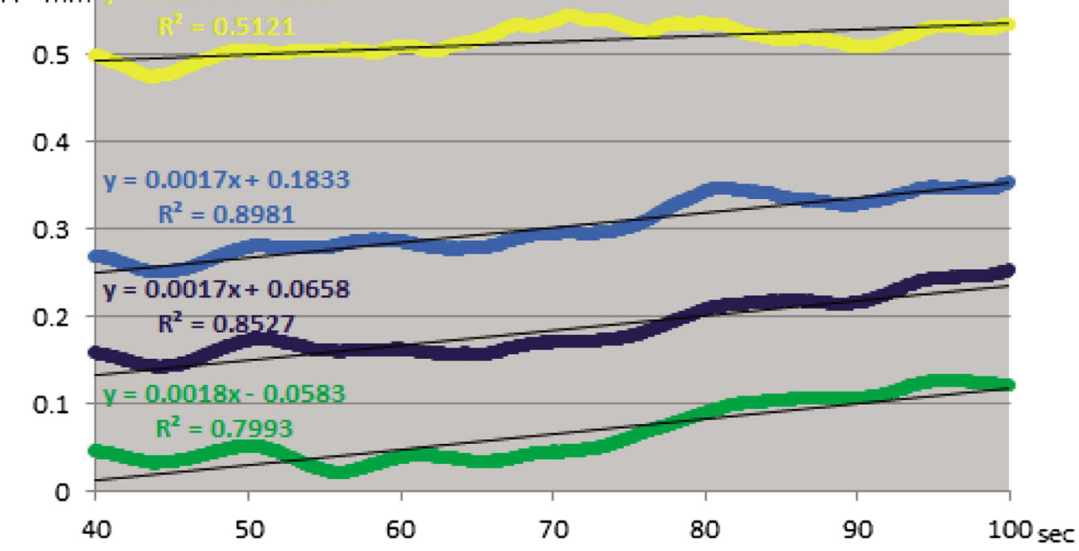

Fig. 4 Representative waveforms for the four clusters segmented into three task periods. a For $5 \mathrm{~s}$ after beginning the Shiritori task. b From 5 to $30 \mathrm{~s}$ after beginning the task. c From $30 \mathrm{~s}$ after beginning to finishing the task. The regression line, regression formula, and coefficient of determination are shown on each waveform. Color coding is the same as in Fig. 3

Figure 4 shows the results of analyzing the characteristics of the changes in [oxy- $\mathrm{Hb}$ ] on the basis of the regression formulas. In the task period from the beginning to $5 \mathrm{~s}$, the four waveforms showed a linear rise (Fig. 4a). The approximate ratio of the slopes of the regression lines was Cluster I: Cluster II: Cluster III: Cluster IV = 10:9:7:4, which 
denotes the ratio of increasing velocity of [oxy-Hb] changes. From $5 \mathrm{~s}$ to $30 \mathrm{~s}$ after beginning the task, only the Cluster I waveform continued to rise without falling. The approximate ratio of the slopes of the regression lines in this period was Cluster I: Cluster II: Cluster III: Cluster IV = 4:2:1:0 (Fig. 4b). From 30 s onwards, all waveforms except Cluster IV showed a gradual rise up to finishing the task, and Cluster II and III waveforms were almost parallel. The approximate ratio of the slopes of the regression lines was Cluster I: Cluster II: Cluster III: Cluster IV =1:2:2:3 (Fig. 4c).

Pearson's correlation coefficients between the mean changes in [oxy- $\mathrm{Hb}]$ and the number of words generated during the Shiritori task revealed no significant correlation.

To determine the cortical laterality of changes in [oxy-Hb] during the Shiritori task, the average of the mean changes in [oxy-Hb] across all subjects was compared for the left and the right hemisphere channels at symmetrical positions using a two-tailed paired Student's $\mathrm{t}$-test. The analysis indicated no significant differences (d.f. $=24, \mathrm{t}=-0.087, p=0.931$ ). Additionally, for each subject, a similar analysis of laterality was conducted on mean changes in [oxy-Hb]. Left hemispheric dominance was found in 6 subjects (FDR: $p=7.04 \mathrm{E}-05$ to 0.008) and right hemispheric dominance was found in 4 subjects (FDR: $p=1.12 \mathrm{E}-05$ to 0.004 ). The other 15 subjects showed no dominance statistically.

\section{Discussion}

In this study we used NIRS, which can cover frontal and superior temporal surface areas, to determine the patterns of cortical activation of healthy subjects performing the Shiritori task with overt speech. We found significant hemodynamic responses bilaterally in $42(80.8 \%)$ of the 52 channels examined. The wide distribution of significantly activated channels across the frontal and superior temporal areas found in this study is in line with the results of previous studies, which determined cortical activation patterns of healthy adults during the Shiritori task without overt speech by using MEG and fMRI, respectively [14, 27].

Using cluster analysis of the changes in [oxy- $\mathrm{Hb}$ ] per $0.1 \mathrm{~s}$, we divided the 52 channels into 4 clusters, each comprised of channels showing similar changes in [oxy- $\mathrm{Hb}$ ] and distributional region symmetrically across hemispheres (Fig. 3). It is speculated that the representative cortical regions in each cluster could be the inferior frontal gyrus and superior temporal gyrus in Cluster I, the middle frontal gyrus in Cluster II, the superior frontal gyrus in Cluster III, and the supplementary motor area in Cluster IV according to $[19,26]$, the former showed the probabilistic correspondence relation between anatomical cortical surface areas and the international 10-20 system for EEG, and the latter used the same NIRS machine (ETG-4000 with probe of 52 channels) and method for setting the probe as our study, and demonstrates a figure to clarify correspondence between the channels and the anatomical areas.

Next, we determined the characteristics of the changes in [oxy-Hb] (waveforms) throughout the task by analyzing an averaged waveform (representative waveform) across the channels included in each cluster. The differences among the representative waveforms were determined from the three successive task periods set: the steep slope of the initial rise (Fig. 4a), the duration of this rise (Fig. 4b), and the subsequent gentle slope up until the end of the task (Fig. 4c). The most highly-activated representative waveform (out of the four waveforms) was that of Cluster I, which was independent from the other three clusters according to the dendrogram derived from cluster 
analysis (Fig. 3). Its initial rise was steeper and lasted longer than that of the others during the first task period, and almost plateaued during the last period. Since Cluster I would mainly include the inferior frontal and post-superior temporal gyri encompassing Broca' $s$ and Wernicke' $s$ areas respectively which constitute language-related networks in the brain [27], the variations seen between the cluster waveforms might reflect strong functional connectivity between the channels in the same cluster [11]. However it is currently not known whether differences between clusters depend on different cognitive components involved in tasks, or whether they reflect specific traits of the neurons comprising the different cortical regions. Applying the present analysis to some cognitive tasks and resting states may help to clarify the situation.

With regard to laterality, it was confirmed that the channels showing significant cortical activation during the Shiritori task were distributed bilaterally, and that this bilateral distribution was found in $60 \%$ of subjects. This result is opposed to the leftsided dominance during the Shiritori tasks reported in MEG and fMRI studies [14, 27]. Although further research is required to determine the cortical laterality during Shiritori, it is important to point out that the duration of the Shiritori task in the present study was longer than that of the other studies in this field. This would increase the cognitive loads of the task, leading to activation of more cortical areas, including regions in the right hemisphere.

Next, with regard to the wide frontal and superior temporal activation found in this study, we attempt to speculate the reason for this activation associated with Shiritori task performance. As with conventional WGL, executive functions such as word retrieval, storing previously generated word in working memory, maintaining cognitive effort, inhibiting inappropriate responses and sufficient processing speed aiming at better performance would be demanded in order to perform the Shiritori task. These functions are primarily subserved by the frontal lobe $[1,10,20]$. The Shiritori task, however, appears to have greater executive demands than the conventional WGL. This is due to the fact that the cognitive loads of set-shifting and inhibition may be higher because of the initial syllable changes for each word and avoiding saying a noun ending with $/ \mathrm{n} /$, respectively. In addition, playing the Shiritori task alone would demand self-monitoring and planning because the subject must think back to the last noun said as well as think ahead when choosing the next noun. Moreover, the Shiritori task is thought to place greater cognitive loads on internal phonological processing in order to repeat and listen to the last syllable of the previous noun in mind until generating the next noun, and the superior temporal activity is considered to be responsible for this function [20,27]. Thus, Shiritori might require further activation of the frontal and superior temporal areas than conventional WGL.

This study has several limitations. First, the cortical region/channel association in the present study differs depending on subject's head shape as the channels depart from Fpz (the international 10/20 system for EEG) at which we set the most ventral center photodiode. Thus, especially in superior temporal areas, a perfect cortical region/channel association would not be ensured. In the future study for the purposes of accurate identification of localization, it is required to investigate the cortical region/channel association in each subject or to register the channel positions onto the standard template brain when group statistics is performed $[25,28]$. Second, changes in [oxy-Hb] are expressed as relative values compared to a baseline task, and it's assumed that 
optical path length is constant across subjects or measurement points. Thus, evaluations using this type of NIRS (continuous wave-based) machine which is unable to measure absolute values should be considered estimates. Nevertheless, it was argued that this kind of NIRS machine is able to measure quite precise changes in [oxy-Hb] as well as [deoxy-Hb] since the path length did not change more than $10 \%$ among channels $[6,7]$. Third, it was recently reported that NIRS signals would include considerable components from skin blood flow, a fact that is attracting the interest of researchers using NIRS [16, 23]. Although this has been confirmed only at the frontal pole (forehead) thus far, clarifying the hemodynamic responses across the whole scalp and seeking methods to minimize the influence of skin blood flow is urgently needed to justify the results determined by NIRS and to ensure NIRS studies continue. However, since a strong correlation between NIRS and fMRI signals has also been reported [3, 21], the activity seen in the present study could also include considerable signals from neural activity. Therefore, at the very least, it appears we succeeded in outlining the cortical activity to perform the Shiritori task. The development of techniques that can separate systemic and brain activity components from NIRS signals is awaited.

\section{Conclusions}

This study determined, using NIRS, the patterns of cortical activation in healthy subjects while performing "Shiritori", a popular Japanese game based on WGL with overt speech. Cortical activation was widely distributed over the bilateral superior temporal cortex as well as the frontal cortex during the task, as is the case in the previous functional neuroimaging studies by MEG and fMRI during the Shiritori task without overt speech. Furthermore, this activation could be divided into four clusters, with the channels in each cluster showing similar hemodynamic responses, one of which appears to correspond to the frontal and temporal language-related networks in the brain. These findings suggest that Shiritori might be used as a frontal and temporal activating WGL with potential clinical implications.

\section{Additional file}

Additional file 1: Grand average waveforms of changes in [oxy-Hb] (red), [deoxy-Hb] (blue) and [total-Hb] (green) during the Shiritori task. For each channel, the $x$-axis denotes time from 0 to $160 \mathrm{~s}$ and the $y$-axis denotes activation between -0.8 and $0.8 \mathrm{mM} \mathrm{mm}$. The Shiritori task period is marked by two vertical dashed lines at 10 and 100 s. CH, channel. (PDF $830 \mathrm{~kb}$ )

\footnotetext{
Abbreviations

[deoxy-Hb]: concentration of deoxygenated hemoglobin; [oxy-Hb]: concentration of oxygenated hemoglobin; FDR: false discovery rate; fMRI: functional magnetic resonance imaging; MEG: magnetoencephalography; NIRS: nearinfrared spectroscopy; WGL: word generation to letters.

Competing interests

The authors declare that they have no competing interests.

Authors' contributions

TN RI and MI conceived and designed the experiments. TN and RI performed the experiments. TN RI and Ml analyzed the data. TN RI HT and YK contributed materials and analysis tools. TN RI LC and MI wrote the paper. Final version of the manuscript was read and approved by all authors.
} 


\section{Author details}

'Department of Clinical Neuroscience and Psychiatry, Osaka University Graduate School of Medicine, D3 2-2 Yamadaoka, Suita, Osaka 565-0871, Japan. ${ }^{2}$ Department of Child and Adolescent Mental Health, National Institute of Mental Health, National Center of Neurology and Psychiatry, 4-1-1 Ogawahigashi-cho, Kodaira, Tokyo 187-8553, Japan. ${ }^{3}$ Laboratory of Cognitive and Computational Neuroscience, Center for Biomedical Technology, Madrid Complutense University (UCM) and Madrid Polytechnic University (UPM), Campus Montegancedo, 28223 Pozuelo de Alarcón, Madrid, Spain.

Received: 2 October 2015 Accepted: 3 January 2016

Published online: 24 January 2016

\section{References}

1. Alvarez JA, Emory E. Executive function and the frontal lobes: a meta-analytic review. Neuropsychol Rev. 2006;16:17-42.

2. Benjamini Y, Hochberg Y. Controlling the false discovery rate: a practical and powerful approach to multiple testing. J R Stat Soc [Ser B]. 1995;57:289-300.

3. Cui X, Bray S, Bryant DM, Glover GH, Reiss AL. A quantitative comparison of NIRS and fMRI across multiple cognitive tasks. Neuroimage. 2011;54:2808-21.

4. $\quad$ Dan I, Singh AK. Exploring the false discovery rate in multichannel NIRS. Neuroimage. 2006;33:542-9.

5. Dieler AC, Tupak SV, Fallgatter AJ. Functional near-infrared spectroscopy for the assessment of speech related tasks. Brain Lang. 2012:121:90-109.

6. Ferrari M, Mottola L, Quaresima V. Principles, techniques, and limitations of near infrared spectroscopy. Can J Appl Physiol. 2004;29:463-87.

7. Ferrari M, Wei Q, Carraresi L, De Blasi RA, Zaccanti G. Time-resolved spectroscopy of the human forearm. J Photochem Photobiol B. 1992;16:141-53.

8. Fox PT, Raichle ME. Focal physiological uncoupling of cerebral blood flow and oxidative metabolism during somatosensory stimulation in human subjects. Proc Natl Acad Sci USA. 1986;83:1140-4.

9. Fujiki R, Morita K, Sato M, Yamashita Y, Kato Y, Ishii Y, et al. Single event-related changes in cerebral oxygenated hemoglobin using word game in schizophrenia. Neuropsychiatr Dis Treat. 2014;10:2353-60.

10. Henry JD, Crawford JR. A meta-analytic review of verbal fluency performance following focal cortical lesions. Neuropsychology. 2004;18:284-95.

11. Homae F, Watanabe H, Otobe T, Nakano T, Go T, Konishi Y, et al. Development of global cortical networks in early infancy. J Neurosci. 2010:30:4877-82.

12. Hoshi Y. Functional near-infrared optical imaging: utility and limitation in human brain mapping Psychophysiology. 2003:40:511-20

13. Hoshi Y, Kobayashi N, Tamura M. Interpretation of near-infrared spectroscopy signals: a study with a newly developed perfused rat brain model. J Appl Physiol. 2001;90:1657-62.

14. Inoue M, Ueno T, Morita K, Shoji Y, Matsuoka T, Fujiki R, et al. Brain activities on fMRI using the shiritori task in normal subjects. Kurume Med J. 2010;57:109-15.

15. Jobsis FF. Noninvasive infrared monitoring of cerebral and myocardial oxygen sufficiency and circulatory parameters. Science. 1997;198:1264-7.

16. Kirilina E, Jelzow A, Heine A, Niessing M, Wabnitz H, Brühl R, et al. The physiological origin of task-evoked systemic artefacts in functional near infrared spectroscopy. Neuroimage. 2012;61:70-81.

17. Nakahachi $T$, Ishii $R$, Iwase $M$, Canuet $L$, Takahashi $H$, Kurimoto $R$, et al. Frontal activity during the digit symbol substitution test determined by multichannel near-infrared spectroscopy. Neuropsychobiology. 2008;57:151-8.

18. Nakahachi $T$, Ishii $R$, Iwase M, Canuet $L$, Takahashi H, Kurimoto $R$, et al. Frontal cortex activation associated with speeded processing of visuospatial working memory revealed by multichannel near-infrared spectroscopy during Advanced Trail Making Test performance. Behav Brain Res. 2010;215:21-7.

19. Okamoto M, Dan H, Sakamoto K, Takeo K, Shimizu K, Kohno S, et al. Three-dimensional probabilistic anatomical cranio-cerebral correlation via the international 10-20 system oriented for transcranial functional brain mapping. Neuroimage. 2004;21:99-111.

20. Price CJ. A review and synthesis of the first 20 years of PET and fMRI studies of heard speech, spoken language and reading. Neuroimage. 2012;62:816-47.

21. Strangman G, Culver JP, Thompson JH, Boas DA. A quantitative comparison of simultaneous BOLD fMRI and NIRS recordings during functional brain activation. Neuroimage. 2002;17:719-31.

22. Suzuki T. Kotoba asobi (Word game). Tokyo: Kodansya Ltd.; 2009. p. 24-33 (in Japanese)

23. Takahashi T, Takikawa Y, Kawagoe R, Shibuya S, Iwano T, Kitazawa S. Influence of skin blood flow on near-infrared spectroscopy signals measured on the forehead during a verbal fluency task. Neuroimage. 2011;57:991-1002.

24. Takizawa R, Kasai K, Kawakubo Y, Marumo K, Kawasaki S, Yamasue H, et al. Reduced frontopolar activation during verbal fluency task in schizophrenia: a multi-channel near-infrared spectroscopy study. Schizophr Res. 2008;99:250-62.

25. Tsuzuki D, Cai DS, Dan H, Kyutoku Y, Fujita A, Watanabe E, et al. Stable and convenient spatial registration of stand-alone NIRS data through anchor-based probabilistic registration. Neurosci Res. 2012;72:163-71.

26. Tupak SV, Badewien M, Dresler T, Hahn T, Ernst LH, Herrmann MJ, et al. Differential prefrontal and frontotemporal oxygenation patterns during phonemic and semantic verbal fluency. Neuropsychologia. 2012;50:1565-9.

27. Yamamoto M, Ukai S, Shinosaki K, Ishii R, Kawaguchi S, Ogawa A, et al. Spatially filtered magnetoencephalographic analysis of cortical oscillatory changes in basic brain rhythms during the Japanese 'Shiritori' Word Generation Task. Neuropsychobiology. 2006;53:215-22.

28. Ye JC, Tak S, Jang KE, Jung J, Jang J. NIRS-SPM: statistical parametric mapping for near-infrared spectroscopy. Neuroimage. 2009;44:428-47. 